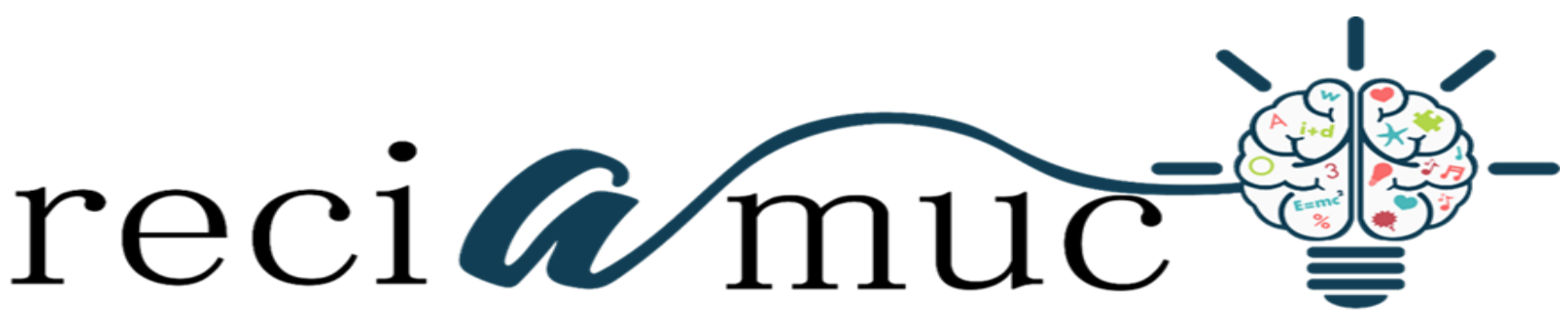

Revista cientifica de investigación actualización del mundo de las ciencias

Erika Teresa Tumbaco Macías a; Johang Mauricio Olivo Román ${ }^{\text {b; }}$ Cesar Enrique Arreaga Pérez ${ }^{c}$; Ronald Adrián Valencia Rodríguez ${ }^{\mathrm{d}}$

Vasculitis de debut en la infancia

Vasculitis's debut in the childhood

Revista Científica de Investigación actualización del mundo de las Ciencias. Vol. 3 núm., 1, enero, ISSN: 2588-0748, 2018, pp. 286-309

DOI: $10.26820 /$ reciamuc/3.(1).enero.2019.286-309

URL: $\underline{\text { htp: }: / / \text { reciamuc.com/index.php/RECIAMUC/article/view/236 }}$

Código UNESCO: 3205 Medicina Interna

Tipo de Investigación: Artículo de Revisión

Editorial Saberes del Conocimiento

Recibido: 10/12/2018

Aceptado: 20/01/2019

Publicado: 30/01/2019

Correspondencia: dircetor@ reciamuc.com
a. Médico; Guayaquil, Ecuador.
b. Médico; Guayaquil, Ecuador.
c. Médico; Guayaquil, Ecuador.
d. Médico; Guayaquil, Ecuador. 


\section{Vasculitis de debut en la infancia}

Vol. 3, núm. 1., (2019)

Erika Teresa Tumbaco Macías; Johang Mauricio Olivo Román; Cesar Enrique Arreaga Pérez; Ronald Adrián Valencia Rodríguez

\section{RESUMEN}

En el campo de las ciencias médicas vinculadas con el área pediátrica se encuentran una gama de enfermedades de interés investigativo, entre ellas: la vasculitis de debut, vista como un proceso patológico caracterizado por inflamación y necrosis de los vasos sanguíneos que provocan isquemia distal a la lesión en los órganos y territorio irrigados por estos vasos. Asimismo, puede afectar vasos de cualquier calibre, incluidos los capilares, vénulas y arterias musculares de pequeño, mediano y gran calibre. La enfermedad comparte algunos signos comunes y las manifestaciones iniciales suelen ser semejantes dependiendo del órgano o sistema afectados. Por ello, los especialista pediátricos realizan un diagnóstico funcional y confiable mediante pruebas histológicas o hagiográfica de la lesión vascular, cuando son afectados grandes vasos, también la biopsia debe tomarse en una zona anormal o patológica o con un tamaño relativamente grande; es decir, aunque existe vasculitis debido a que presentan manifestaciones clásicas es posible el diagnóstico basándose solo en datos clínicos. Ante esto, su tratamiento se hace en función al cuadro clínico que presenta e intensidad, si es un proceso localizado o un cuadro generalizado. Para, La Cruz y Mir (2014), "la mayoría de las vasculitis primarias son raras en la infancia, pero se asociación con una morbilidad y mortalidad significativa, las más frecuentes son la púrpura de Schonlein -Henoch y enfermedad de Kawasaki". (p.12). Este planteamiento, lleva a entender que la presencia de la enfermedad durante la infancia registra una morbilidad significa, en otras palabras, se encuentra un número significativo de niños o niñas que padecen la enfermedad y dan como resultado elevadas defunción de este grupo etario. Consideraciones generales que sirven para el desarrollo del presente artículo que busca analizar la vasculitis debut en la infancia y ofrecer otras apreciaciones para generar nuevas ideas que sirvan de apoyo a las ciencias médicas.

Palabras Claves: Vasculitis Debut; Infancia; Lesión en los Órganos. 


\title{
Vasculitis de debut en la infancia
}

Vol. 3, núm. 1., (2019)

Erika Teresa Tumbaco Macías; Johang Mauricio Olivo Román; Cesar Enrique Arreaga Pérez; Ronald Adrián Valencia Rodríguez

\begin{abstract}
In the field of medical sciences related to pediatric area are a range of investigative interest, including: vasculitis debut, seen as a pathological process characterized by inflammation and necrosis of the blood vessels causing ischemia distal to the lesion in the organs and territory irrigated by these vessels. It can also affect any caliber vessels, including the capillaries, venules and muscular arteries of small, medium and large caliber. The disease shares some common signs and the initial manifestations tend to be similar depending on the organ or system concerned. Therefore, the Pediatric specialist carried out a functional and reliable diagnosis using histological tests or angiography of vascular injury, when large vessels are affected, also the biopsy should be in an abnormal or pathological area or with a size relatively large; Although there is vasculitis since they present classic manifestations i.e. possible diagnosis based only on clinical data. Given this, your treatment is made according to the clinical picture that presents and intensity, if it is a localized process or a generalized picture. For, La Cruz and Mir (2014), "the majority of primary vasculitis are rare in children, but is association with morbidity and significant mortality, the most frequent are la púrpura de Schonlein - Henoch and Kawasaki disease". (p.12). This approach, leads to understand that the presence of the disease during childhood records morbidity means, in other words, is a significant number of children who suffer from the disease, resulting in high death for this age group. General considerations that serve for the development of this article that seeks to analyze debut in childhood vasculitis and provide other insights to generate new ideas that support to medical science.
\end{abstract}

Key Words: Vasculitis Debut; Childhood; Injury to the organs. 


\section{Vasculitis de debut en la infancia}

Vol. 3, núm. 1., (2019)

Erika Teresa Tumbaco Macías; Johang Mauricio Olivo Román; Cesar Enrique Arreaga Pérez; Ronald Adrián Valencia Rodríguez

\section{Introducción.}

La infancia como etapa del desarrollo humano se inicia desde el nacimiento y culmina en la pubertad; por lo tanto, en ella se incluyen todos los niños y niñas que conforman dicho grupo etario. Sin embargo, durante su crecimiento se presentan diferentes tipos de enfermedades, como la vasculitis, que comprende un grupo heterogéneo de entidades relativamente frecuentes (suponen 1 de cada 300 ingresos en un hospital universitario) de etiologías y manifestaciones diversas, caracterizado por la inflamación de los vasos sanguíneos, arterias, venas o ambos, lo que compromete su función con el desarrollo de isquemia y necrosis.

En consecuencia, la inflamación vascular puede acompañarse de una sintomatología general (fiebre, astenia, afectación del estado general) y desarrollo de manifestaciones locales orgánicas de acuerdo al órgano afecto por la vasculitis (afectación cutánea, síntomas neurológicos, dolor abdominal, compromiso renal, entre otras.). De igual manera, la piel y el tejido subcutáneo son afectan frecuentemente en las vasculitis, probablemente se deba a diversos factores como: el gran número de vasos dérmicos, exposición al frio y la presencia de fenómenos de estasis vascular que favorecerían el desarrollo de vasculitis con afectación cutánea.

En este orden de ideas, puede destacar que la vasculitisa nivel cutáneo puede manifestarse de diversas formas, desde cambios en la coloración, edema, púrpura, equimosis y necrosis (úlceras), destacándose entre ella, con mayor frecuencia el desarrollo de púrpura palpable en extremidades inferiores. No obstante, la presencia de una púrpura palpable puede ser una manifestación de una vasculitis benigna de corta duración, puramente cutánea; en cambio una 


\section{Vasculitis de debut en la infancia}

Vol. 3, núm. 1., (2019)

Erika Teresa Tumbaco Macías; Johang Mauricio Olivo Román; Cesar Enrique Arreaga Pérez; Ronald Adrián Valencia Rodríguez

manifestación cutánea de una vasculitis sistémica puede acompañarse de afectación orgánica con compromiso vital. En tal sentido, La Cruz y Mir (ob.cit), precisan que una "elevada frecuencia de manifestaciones cutáneas de las vasculitis hace que el reconocimiento de estas lesiones, sea importante para el diagnóstico de las mismas"(p. 18).

De las evidencias anteriores, se puede indicar que la presencia de la vasculitis como enfermedad que afecta los vasos, la piel, compromiso renal y manifestaciones locales entre otros, precisa que los médicos pediátricos durante la atención de los niños y niñas que acuden a la consulta, deben mediante la realización de diversos exámenes de laboratorio, que le proporcionen información y datos significativos para llevar a indicar la sospecha clínica de la enfermedad. Por lo tanto, deben recomendar exámenes tales como: velocidad de sedimentación (VSG), o el aumento de la proteína C reactiva (PCR), aparición de una anemia. Asimismo, existen unas pruebas generales:(hemograma completo, VSG, PCR), y otras específicas cuando hay afectación de un órgano determinado, como: creatinina, urea, transaminasas, ANA, antiDNA, anti-Sm, Anti-Ro/La, ANCA, anti-cuerpo contra la hepatitis B, complemento (C3 y C4), factor reumatoide, crioglobulinas y enzima conversora de la angiotensina.

Cabe agregar que cada una de las pruebas citadas, deben ser complementadas con la realización de estudios de electromiografía y conducción de nervios, electrocardiograma o ecocardiograma, ecografía, arteriografías, radiografía de tórax o la resonancia nuclearmagnética. Pero a pesar de todas estas pruebas, la que proporciona el diagnóstico absoluto de vasculitis es la biopsia de los tejidos afectados. Pero no siempre es posible obtener muestras para biopsia. Según Quintana (2015) la vasculitis “reúne a un grupo de enfermedades clínicamente heterogéneas con 


\section{Vasculitis de debut en la infancia}

Vol. 3, núm. 1., (2019)

Erika Teresa Tumbaco Macías; Johang Mauricio Olivo Román; Cesar Enrique Arreaga Pérez; Ronald Adrián Valencia Rodríguez

un sustrato histológico común, es decir, presencia de infiltrado inflamatorio en el espesor de la pared de los vasos.”(p.125)

Dentro de este marco de ideas, la presencia de la vasculitis durante la infancia como resultado a ser una enfermedad, con características diferentes entre los pacientes, requiere de una actuación precisa y coherente del médico para estimar su respectivo diagnóstico. Además, este cambio patológico, localización de la región, tamaño del vaso y extensión sistémica del daño vascular, se convierten en expresiones clínicas encargadas de lograr con severidad de su presencia en el paciente. Se hace necesario resaltar, que las lesiones cutáneas a menudo son semejantes en enfermedades distintas, si bien el tipo de ellas (como serían púrpura, nódulo o gangrena) puede aportar una pista en cuanto al tipo o diámetro del vaso afectado.

En consecuencia, la piel por ser un órgano importante, que se afecta en muchos síndromes vasculíticos como primera manifestación, por tal motivo sirve de orientación diagnóstica. Según, González (2015) destaca que "es muy difícil pasar a especificar unos síntomas generales que en ocasiones van a ser inespecíficos, como pueden ser la fiebre, astenia, anorexia, pérdida de peso, debilidad o fatiga."(p.36). De acuerdo con este planteamiento, el diagnóstico preciso en cuanto a la presencia de la vasculitis, se presenta para el médico tratante en una intervención acorde para poder llegar a indicar que existe la presencia de la enfermedad, de lo contrario, puede indicar apreciaciones relativas a otro proceso que este caracterizado con algunas de las manifestaciones de la vasculitis.

Asimismo, González (2015) plantea que es importante indicar a los padres que la vasculitis "es un proceso que puede ser infeccioso e inmunológico y que se trata de lesiones 


\section{Vasculitis de debut en la infancia}

Vol. 3, núm. 1., (2019)

Erika Teresa Tumbaco Macías; Johang Mauricio Olivo Román; Cesar Enrique Arreaga Pérez; Ronald Adrián Valencia Rodríguez

localizadas en los vasos sanguíneos, cuyo pronóstico y evolución dependerán del órgano que se afecte". (p.51). Lo citado, permite resaltar que cuando los pediatras como especialista se encuentran ante la presencia de un caso de vasculitis, no pueden fijar posiciones directas y abiertas hacia los padres o madres, pues, las condiciones histológica de la enfermedad puede llevarlo hacia una equivocación, situación que generaría consecuencias negativas para el grupo familiar; por ello, es necesario cumplir con los diferentes procesos de diagnósticos para tener certeza de dicha enfermedad.

Igualmente en este proceso diagnóstico el pediatra, se puede encontrar con otras manifestaciones como: artralgias, mialgias o artritis; además, de existir afectación renal y por consiguiente síntomas renales que pueden ocurrir en nefritis, hipertensión arterial o bien un infarto renal. Igualmente en el pulmón se puede encontrar hemorragias pulmonares, nódulos, infiltrados o cavidades. Asimismo, la presencia de sinusitis, otitis, iritis o condritis. Estos síntomas se refieren al contexto general del proceso, pero el clínico debe buscar la combinación de estos signos para plantear el diagnóstico de presunción. Por ello, el tratamiento debe estar ajustado a las condiciones generales que caracterizan dicha enfermedad y así iniciar un proceso que estime los respectivos cambios positivos en los niños y niñas afectados.

De las evidencias anteriores, se puede indicar que las mismas resultan un aporte significativo para el desarrollo de este artículo, pues, es relevante ante de llegar a un diagnóstico de la vasculitis durante la infancia, la confirmación de una suma de exámenes y evaluaciones encargadas de ofrecer al médico especialista valores o hallazgos precisos que le faciliten la respectiva estimación de la enfermedad. Es decir, para poder ofrecer a los padres y madres las 


\section{Vasculitis de debut en la infancia}

Vol. 3, núm. 1., (2019)

Erika Teresa Tumbaco Macías; Johang Mauricio Olivo Román; Cesar Enrique Arreaga Pérez; Ronald Adrián Valencia Rodríguez

orientaciones pertinentes que le servirán de referencia para entender la enfermedad y actuar correctamente para así alcanzar la mejoría que el niño o niña amerita.

Las afirmaciones expuestas, son las encargadas de lograr el desarrollo analítico que caracteriza al presente artículo, su direccionalidad científica hará posible encaminar el proceso hacia la búsqueda de informaciones precisas para ordenarlas y llegar a indicar un conjunto de conclusiones generales que servirán de apoyo en la dinámica médica relacionada con la vasculitis debut en la infancia.

\section{Método.}

Los aspectos que conforman este apartado se encuentran determinados por una serie de elementos dirigidos a reconocer el camino metodológico del estudio, para así especificar sus características generales y fijar posiciones claras en cuanto al método, tipo de investigación, fuentes documentales y técnicas de recolección de información. En cuanto al método, se selecciona inductivo, definido por Méndez (2018) “es el proceso de razonamiento de una parte de un todo, va de lo particular a lo general, de lo individual a lo universal". (p.44). Es decir, mediante la incorporación de este método, se logra desglosar en forma individual los aspectos que conforman el tema seleccionado, para luego agrupar las ideas y formular acciones innovadoras que aseguren interpretaciones válidas para todo los lectores.

Según la definición dada, se puede considerar que el desarrollo del artículo amerita, de separar las consideraciones para convertirlas en acciones reales que puedan ser comprendidas por diferentes grupos interesados en la temática y así, asegurar su expansión en el campo de las 


\section{Vasculitis de debut en la infancia}

Vol. 3, núm. 1., (2019)

Erika Teresa Tumbaco Macías; Johang Mauricio Olivo Román; Cesar Enrique Arreaga Pérez; Ronald Adrián Valencia Rodríguez

ciencias médicas, ambientes familiares $\mathrm{u}$ otros grupos, interesados por descifrar algunas interrogantes relativas a la enfermedad de la vasculitis para tomar posiciones viables.

\section{Tipo de Investigación}

El desarrollo de la presente investigación, corresponde al tipo documental, definida por Mogollón (2019) como aquella que está "basada en nuevos conocimientos con el objetivo de aumentar los ya existentes sin utilizar aplicaciones prácticas, sólo documentaciones que sirva como material de estudio". (p.64). Por ello, su conducción a través de este tipo de estudio, podrá hacer posible dirigir un proceso de revisión en diferentes materiales impresos, tecnológicos que permitan con el apoyo del método inductivo, individualizar los criterios para luego convertirlos en hechos universales.

Otra definición considerada para argumentar el presente artículo, son el ofrecido por, Castellanos (2018) "mediante la investigación documental se puede organizar de forma flexible diferentes criterios establecidos y convertirlos en nuevas directrices para propiciar verdaderos cambios en un documento científico e interpretativo". (p.32). Es decir, el adecuar los pasos que estructuran el camino metodológico del proceso científico, significa ubicar el trabajo dentro de una conceptualización que ofrezca nuevas forma de direccionar el hacer, en otras palabras, ayudar al investigador a construir sus ideas tomando como punto de referencia los aportes de otros criterios. 


\section{Vasculitis de debut en la infancia}

Vol. 3, núm. 1., (2019)

Erika Teresa Tumbaco Macías; Johang Mauricio Olivo Román; Cesar Enrique Arreaga Pérez; Ronald Adrián Valencia Rodríguez

\section{Fuentes Documentales}

Con el fin de dar continuidad al proceso investigativo, es importante ubicarse en aquellas fuentes documentales que sirven de aporte para la ubicación y selección del material impreso y tecnológico vinculado con el tema previamente seleccionado, Por ello, para cumplir dichas actividades se tuvo la necesidad de implementar métodos ajustados a las condiciones documentales que caracterizan al trabajo, dentro de ellos, se incluyen el deductivo mediante el cual, se lograron interpretar las ideas encontrada en los textos o material bibliográfico desde una visión general, para llegar a establecer argumentaciones parciales o particulares. De este modo, se fija su correspondencia con lo señalado por, Castellanos (ob.cit) "representadas por aquellos informes transcritos que sirven para ampliar de manera precisa el contenido de un tema." (p.41).

En consecuencia su utilización permite llegar a la revisión de la diferentes artículos, revistas, libros entre otros que responde a la naturaleza de la temática en el proceso investigativo, aunado a la complementación que dan los diversos textos médicos para ampliar las apreciaciones pertinentes. Tal como lo indica, Castellanos (ob.cit), "representan los aportes bibliográficos para el desarrollo de la temática previamente seleccionada y transformarla en un acto investigativo real". (p.74)

\section{Técnicas para la Recolección de la Información}

En cuanto a las técnicas empleadas se destacan el fichaje mediante el cual, se organizaron los diversos textos de acuerdo a la necesidad de las fuentes primarias requeridas para el desarrollo de los contenidos a tratar en la investigación. Otra técnica de importancia fue el resumen, mediante ella, se desarrollaron aquellos elementos particulares extraídos de textos 


\section{Vasculitis de debut en la infancia}

Vol. 3, núm. 1., (2019)

Erika Teresa Tumbaco Macías; Johang Mauricio Olivo Román; Cesar Enrique Arreaga Pérez; Ronald Adrián Valencia Rodríguez

generales que luego al ser incorporados al argumento general del estudio, hicieron posible su complementación teórica. Cada uno de los pasos fijados, llevan a resaltar que el análisis correspondiente se cumple, mediante la introducción de aquellos métodos descriptivos e interpretativos, capaces de llevar a la autora hacia la construcción de diferentes argumentos consistentes en sus valoraciones como resultado a la lectura previa de los documentos requeridos para el desarrollo del proceso investigativo.

Cabe agregar, que cada uno de los elementos incorporados en el proceso de indagación, hacen posible abrir paso a las primeras actividades inherentes a la selección del tema, revisión de la literatura pertinente, para luego organizar, subrayar e interpretar sus contenidos de manera reflexiva y hacer de ello aportes relevantes que dan cabida a nuevas valoraciones ideales estrechamente relacionadas con el contenido temático, para asegurar la efectividad del mismo al momento de su inclusión en el campo de las ciencias médicas. Es decir, el trabajo científico para obtener resultados significativos, debe ser guiado por el desarrollo de técnicas básicas que aseguren con anticipación la construcción de un reportaje eficiente para todos los lectores interesados en su contenido.

\section{Resultados.}

Los contenidos que sirven de referencia teórica al presente artículo, son vistos como herramientas básicas encargadas de ayudar a fortalecer nuevas interpretaciones desde una perspectiva inductiva, que dará como expresión la valoración y representación de aquellas ideas expuestas previamente $\mathrm{y}$ fueron disgregadas para convertirlas en apreciaciones generales proyectadas hacia un contexto diferente. 


\section{Vasculitis de debut en la infancia}

Vol. 3, núm. 1., (2019)

Erika Teresa Tumbaco Macías; Johang Mauricio Olivo Román; Cesar Enrique Arreaga Pérez; Ronald Adrián Valencia Rodríguez

\section{Vasculitis, Patogénesis y Clasificación}

La presencia de la vasculitis como se indicará en textos anteriores, es una enfermedad que en la infancia para ser diagnosticada requiere de una serie de exámenes de laboratorio y radiografía, hasta llegar a efectuar la biopsia, tomando en cuenta que la piel u órgano afectado tenga condiciones para tomar la muestra. Debido que, las vasculitis pueden manifestarse a nivel cutáneo de diversas formas, desde cambios en la coloración, edema, púrpura, equímosis y necrosis (úlceras), siendo la manifestación más frecuente el desarrollo de púrpura palpable en extremidades inferiores.

Por ello, su diagnóstico y clasificación se basa especialmente en los mecanismos patogénicos que las producen. Se pueden clasificar las vasculitis en base a los mecanismos de producción que incluyen: la infección directa de los vasos, mecanismos inmunes y vasculitis de causa desconocida. La mayor parte de vasculitis pueden catalogarse dentro de las causas infecciosas o en las inmunológicas. Dado que el tratamiento de las vasculitis infecciosas es radicalmente diferente del de las vasculitis mediadas por daño inmunológico, es importante realizar la distinción entre las dos formas de vasculitis en las fases iniciales de la valoración de estos enfermos. Es importante descartar una causa infecciosa antes de instaurar un tratamiento inmunosupresor. La mayoría de las vasculitis están mediadas por mecanismos inmunes, y se clasifican según los 4 tipos de reacción de hipersensibilidad de Gell y Coombs, propuesta por, Medina (2014) entre ellas destaca: "infección directa de los vasos, inmunológico tipo, I, II, II y IV, así como otras causas”(p.235) 


\section{Vasculitis de debut en la infancia}

Vol. 3, núm. 1., (2019)

Erika Teresa Tumbaco Macías; Johang Mauricio Olivo Román; Cesar Enrique Arreaga Pérez; Ronald Adrián Valencia Rodríguez

\section{Cuadro $N^{\circ} 1$ Clasificación Vasculitis por reacción de Hipersensibilidad}

1. Infección directa de los vasos

2. Inmunológico

1. Tipo I ( IgE)

2. Tipo II (citotóxico)

3. Tipo III (Inmunocomplejos)

4. Tipo IV (medida por linfocitos T)

3. Otras causas

1. Neoplásica

2. Desconocida

Fuente: Medina (ob.cit)

Tipo I (vasculitis alérgica o anafiláctica: incluye las vasculitis asociadas a estados atópicos, urticaria vasculitis y síndromede Churg-Strauss. Se caracterizan por la presencia de niveles séricos y tisulares de IgE elevados. En la fase vasculítica se caracteriza por infiltración angiocéntrica de los vasos por eosinófilos.

Tipo II (citotóxica o citolítica)

a. vasculitis mediadas por ANCA (granulomatosis de Wegener, poliangeitis microscópica y síndrome de Churg-strauss). Los ANCA (anticuerpos anticitoplasma de neutrófilos) son capaces de activar los neutrófilos y las células endoteliales, así como inducir la apoptosis acelerada de los neutrófilos. 


\section{Vasculitis de debut en la infancia}

Vol. 3, núm. 1., (2019)

Erika Teresa Tumbaco Macías; Johang Mauricio Olivo Román; Cesar Enrique Arreaga Pérez; Ronald Adrián Valencia Rodríguez

b. Anticuerpos anti-células endoteliales endoteliales (AECA). Los AECA pueden causar vasculitis por daño directo o por activación del complemento, están involucrados en la enfermedad de Behçet y la enfermedad de Takayasu y tienen especificidad por diferentes regiones vasculares, afectando a vasos de pequeño tamaño en la enfermedad de Behçet y vasos de gran tamaño en la enfermedad de Takayasu.

Tipo III (mediada por inmunocomplejos): el depósito de inmunocomplejos da lugar a la activación del complemento y liberación de los componentes C3 y C5, que producen quimiotáxis de neutrófilos y liberación de enzimas proteolíticas que dañan la pared vascular. Es el grupo más amplio de vasculitis entre las que encontramos la vasculitis leucocitoclásticacutánea, el síndrome de Schonlein-Henoch y poliarteritisnodosa, entre otras formas.

Tipo IV (citotóxica) vasculitis mediada por linfocitos T: en este grupo se incluyen aquellas vasculitis granulomatosas que se caracterizan por la presencia en la pared de los vasos de infiltrados inducidos por linfocitos $T$, especialmente $T_{h} 1$, que serían responsables por medio de la producción de interferon- $\gamma$, de la acumulación de macrófagos que fagocitarían las fibras elásticas. En este grupo de vasculitis se encuentra la arteritis de la temporal.

Según lo planteado anteriormente, se puede decir que la existencia de los diferentes tipos de vasculitis, permiten encontrar una actuación en los vasos sanguíneos, que aumentan su volumen; además, aumenta el depósito de los inmunes complejos que conducen a la utilización de los corticoides como tratamiento inicial y único en una serie de procesos en pediatría, principalmente la complicación digestiva y renal de la púrpura de Schönlein-Henoch, la PAN sistémica infantil, formas leves de vasculitis inespecíficas que se pueden tratar con ciclos breves 


\section{Vasculitis de debut en la infancia}

Vol. 3, núm. 1., (2019)

Erika Teresa Tumbaco Macías; Johang Mauricio Olivo Román; Cesar Enrique Arreaga Pérez; Ronald Adrián Valencia Rodríguez

de glucocorticoides durante cortos periodos de tiempo (una vez descartadas las causas infecciosas).

A continuación se muestra el cuadro $\mathrm{n}^{\circ} 2$ vinculado con la presencia de las vasculitis relacionadas con el tamaño de los vasos sanguíneos con el fin de apreciar la actuación de cada uno las enfermedades presente.

Cuadro $N^{\circ}$ 2. Clasificación de la Vasculitis según el tamaño del Vaso Sanguíneo

\begin{tabular}{|c|c|c|c|}
\hline $\begin{array}{l}\text { Vasculitis } \\
\text { predominantement } \\
\text { e de grandes vasos }\end{array}$ & $\begin{array}{l}\text { Vasculitis } \\
\text { predominantement } \\
\text { e de mediano } \\
\text { vasos }\end{array}$ & $\begin{array}{l}\text { Vasculitis predominantemente de } \\
\text { pequeños vasos }\end{array}$ & Otras vasculitis \\
\hline $\begin{array}{ll}\text { Arteritis } & \text { de } \\
\text { Takayasu } & \end{array}$ & $\begin{array}{l}\text { PoliarteritisNodos } \\
\text { a Infantil } \\
\text { Poliarteritis } \\
\text { Cutánea } \\
\text { Enfermedad de } \\
\text { Kawasaki }\end{array}$ & $\begin{array}{l}\text { Granulomatosas } \\
\text { Granulomatosis de Wegener } \\
\text { Sindrome de Churg Strauss } \\
\text { No granulomatosas } \\
\text { PoliangeitisMicroscopica } \\
\text { Purpura ShonleinHenoch } \\
\text { Vasculitis } \\
\text { Leucocitoclastica cutánea aislada } \\
\text { Vasculitis } \\
\text { urticariformehipocomplementemi } \\
\text { ca }\end{array}$ & $\begin{array}{l}\text { Enfermedad de } \\
\text { Behcet } \\
\text { Vasculitis } \\
\text { secundaria a } \\
\text { infecciones, } \\
\text { tumores } \\
\text { malignos } \\
\text { drogas } \\
\text { incluyendo } \\
\text { vasculitis por } \\
\text { hipersensibilida } \\
\text { d } \\
\text { Vasculitis } \\
\text { asociada } \\
\text { enfermedades } \\
\text { de } \\
\text { conectivo tejido } \\
\text { Vasculitis } \\
\text { aislada del } \\
\text { sistema } \\
\text { nervioso central } \\
\text { Sin clasificar. }\end{array}$ \\
\hline
\end{tabular}

Fuente: Quintana (ob.cit) 


\section{Vasculitis de debut en la infancia}

Vol. 3, núm. 1., (2019)

Erika Teresa Tumbaco Macías; Johang Mauricio Olivo Román; Cesar Enrique Arreaga Pérez; Ronald Adrián Valencia Rodríguez

Al observar el presente cuadro, se evidencia que la existencia de las vasculitis, de acuerdo con el tamaño que caracteriza al vaso sanguíneo, muestra una serie de enfermedades asociadas a los tejidos conectivos, donde la infección es característico y afecta el sistema nervioso central; además conduce a la presencia de tumores malignos u otras enfermedades que amerita una atención particular con el fin de lograr el respectivo abordaje de la sintomatología en el niño o niña.

Cabe agregar, que la vasculitis de vasos pequeñosaproximadamente un $5 \%$ de pacientes que se presentan con vasculitis cutánea tiene una vasculitis sistémica y alrededor de un $3 \%$ tienen una vasculitis sistémica asociada a ANCA. Estas formas de vasculitis constituyen un grupo de enfermedades afectando a vasos de pequeño y mediano calibre que incluyen la granulomatosis de Wegener, la poliangeitis microscópica y el Síndrome de Churg-Strauss. Los ANCA son anticuerpos dirigidos contra antígenos de los polimorfonucleares. Existen 2 patrones de ANCAS: el patrón citoplasmático que incluye los anticuerpos contra la proteinasa 3, y el patron periférico dirigido contra la mieloperoxidasa.

En la actualidad se piensa que ciertas moléculas proinflamatorias como el TNF- $\alpha$ y la IL1 inducen la translocación de la proteinasa 3 y la mieloperoxidasa hacia la superficie de los neutrófilos. Estos antígenos se unen a los ANCA, activando a los neutrofilos y aumentando su adherencia a las células endoteliales dando lugar al daño vascular granular citoplásmico (CANCA, PR3-ANCA, con especificidad ante el antígeno citoplásmicoproteinasa 3). Patrón perinuclear (p-ANCA, MPO-ANCA, con especificidad contra el antígeno mieloperoxidasa) 


\section{Vasculitis de debut en la infancia}

Vol. 3, núm. 1., (2019)

Erika Teresa Tumbaco Macías; Johang Mauricio Olivo Román; Cesar Enrique Arreaga Pérez; Ronald Adrián Valencia Rodríguez

Además, el Granulomatosis con poliangeitis (antes vasculitis de Wegener): Es una vasculitis sistémica primaria caracterizada por la triada clínica devasculitis granulomatosadel tracto superior e inferior, glomerulonefritis y grados variables de vasculitis de pequeño vaso. Los pacientes con GW generalmente presentan síntomas de vías respiratorias superiores, incluyendo sinusitis, obstrucción y perforación nasal que puede dar lugar a una deformidad en silla de montar. Otros síntomas frecuentes incluyen otitis media, dolor de oído y disminución de la capacidad auditiva. La afectación pulmonar puede manifestarse en forma de tos productiva y hemoptisis o puede ser asintomática. La afectación renal en forma de glomerulonefritis determina el pronóstico de los enfermos.

Al menos el 50\% de los pacientes tienen afectación mucocutánea que puede ser la forma de presentación hasta en un 12\% de casos. Las manifestaciones cutáneas pueden ser de 3 tipos: 1)púrpura palpable como manifestación de una vasculitis leucocitoclástica de pequeño vaso, 2) nódulos subcutáneos y úlceras como manifestación de una vasculitis de mediano vaso y 3) lesiones polimorfas que incluyen pápulas y nódulos necróticos en las áreas periarticulares, úlceras a tipo pioderma gangrenoso y lesiones de hiperplasia gingival granulomatosa. La afectación cutánea en la granulomatosis de Wegener se asocia con afectación sistémica activa y progresiva.

Los criterios para el diagnóstico de la GW están resumidos en el cuadro 3 . La granulomatosis de Wegener se asocia de forma específica con la presencia de ANCA con especificidad contra la proteinasa 3, con patrón citoplásmico en los pacientes con enfermedad activa tiene una alta sensibilidad y especificad. En cuanto a la Vasculitis granulomatosa y alérgica de Churg-Strauss: La enfermedad de Churg-Strauss es una vasculitis sistémica primaria 


\section{Vasculitis de debut en la infancia}

Vol. 3, núm. 1., (2019)

Erika Teresa Tumbaco Macías; Johang Mauricio Olivo Román; Cesar Enrique Arreaga Pérez; Ronald Adrián Valencia Rodríguez

rara que se observa en pacientes con asma. Se caracteriza por afectar a vasos de tamaños variables con formación de granulomas intra y extravasculares con intensa presencia de eosinófilos en el infiltrado- y por afectar a pacientes con historia de asma, atopia y eosinofilia periférica. El síndrome de Churg-strauss está considerado resultado de una reacción de hipersensibilidad tipo I, en el cual la proliferación de linfocitos CD4+, TH2 estimulada por diversos alergenos -inhalados, vacunas, medicaciones o infecciones. Los linfocitos TH2 producen interleucina 4, 5 y 13 que estimulan la acumulación de mastocitos, basófilos y especialmente eosinófilos que producen el daño tisular

\section{Cuadro $N^{\circ} 3$ Criterios de Diagnóstico de la Gramulomatosis de Wegener}

Inflamación nasal u oral: úlceras orales

Alteraciones de la radiografía de tórax: nódulos, cavitación o infiltrados no migratorios ni fugaces.

Alteraciones en el sedimento urinario: micro hematuria (de 5 hematíes por campo) o cilindros

Presencia de inflamación granulomatosa en la biopsia

Fuente: Quintana (ob.cit)

Al continuar con la clasificación de las vasculitis es importante destacar aquellas manifestaciones relacionadas con el Sindrome de Churg, están resumidos en el cuadro 4 que se presenta a continuación: 


\section{Vasculitis de debut en la infancia}

Vol. 3, núm. 1., (2019)

Erika Teresa Tumbaco Macías; Johang Mauricio Olivo Román; Cesar Enrique Arreaga Pérez; Ronald Adrián Valencia Rodríguez

\section{Cuadro $N^{\circ}$ 4. Criterios de Diagnóstico Sindrome de Churg}

\begin{tabular}{|l|}
\hline Asma \\
\hline Eosinofilia con $10 \%$ del recuento leucocitario. \\
\hline Mononeuropatía, mononeuritis múltiple o polineuropatía \\
\hline $\begin{array}{l}\text { Infiltrados pulmonares fugaces; Historia de sinusitis aguda o crónica o veladura } \\
\text { radiológica de los senos paranasales;Presencia de infiltración eosinofila extravascular }\end{array}$ \\
\hline
\end{tabular}

Fuente: Quintana (ob.cit)

La enfermedad de Churg-Strauss es una vasculitis sistémica primaria rara que se observa en pacientes con asma. Se caracteriza por afectar a vasos de tamaños variables con formación de granulomas intra y extravasculares -con intensa presencia de eosinófilos en el infiltrado- y por afectar a pacientes con historia de asma, atopia y eosinofilia periférica. El síndrome de Churgstrauss está considerado resultado de una reacción de hipersensibilidad tipo I, en el cual la proliferación de linfocitos CD4+, TH2 estimulada por diversos alergenos -inhalados, vacunas, medicaciones o infecciones. Los linfocitos TH2 producen interleucina 4, 5 y 13 que estimulan la acumulación de mastocitos, basófilos y especialmente eosinófilos que producen el daño tisular

Suele asociar sintomatología sistémica con fiebre, mal estado general y pérdida de peso. La manifestación clínica principal e inicial es el desarrollo de asma, que suele afectar a personas con antecedentes de atopia (bronquitis asmática, rinitis o conjuntivitis alérgica y dermatitis). En un segundo estadio las crisis asmáticas se agravan y suelen acompañarse de infiltrados pulmonares con alteraciones radiológicas y eosinofilia periférica y en un tercer estadio se desarrollan signos y síntomas propios de una vasculitis, solo en este último estadio es posible realizar el diagnóstico del síndrome. El 50\% de los pacientes fallecen por afectación cardíaca de la enfermedad. El $70 \%$ de los pacientes desarrollan lesiones cutáneas en forma de nódulos 


\section{Vasculitis de debut en la infancia}

Vol. 3, núm. 1., (2019)

Erika Teresa Tumbaco Macías; Johang Mauricio Olivo Román; Cesar Enrique Arreaga Pérez; Ronald Adrián Valencia Rodríguez

cutáneos y subcutáneos, denominados granulomas extravasculares de Churg-Strauss, que pueden observarse tanto en la vasculitis de Churg Strauss como en otras enfermedades reumáticas. Otras lesiones cutáneas incluyen el desarrollo de púrpura palpable y livedoreticularis. El 75\% de los pacientes desarrollan manifestaciones neurológicas. La analítica más característica muestra una eosinofília periférica con valores de entre 5 y 10x109 eosinófilos/L. El nivel de eosinofilia es un buen marcador para el tratamiento de la enfermedad. El diagnostico de vasculitis puede sugerirse ante la presencia de asma, eosinofilia, infiltrados pulmonares y datos de afectación multisistémica en pacientes con antecedentes atópicos.

Suele asociar sintomatología sistémica con fiebre, mal estado general y pérdida de peso. La manifestación clínica principal e inicial es el desarrollo de asma, que suele afectar a personas con antecedentes de atopia (bronquitis asmática, rinitis o conjuntivitis alérgica y dermatitis). En un segundo estadio las crisis asmáticas se agravan y suelen acompañarse de infiltrados pulmonares con alteraciones radiológicas y eosinofilia periférica y en un tercer estadio se desarrollan signos y síntomas propios de una vasculitis, solo en este último estadio es posible realizar el diagnóstico del síndrome.

Al hacer referencia a la Poliangeitis microscópica: Forma de vasculitis de pequeño vaso que se asocia frecuentemente a enfermedad renal rápidamente progresiva (glomerulonefritis necrotizante segmentaria focal), afectación cutánea (>75\% púrpura palpable), presencia de anticuerpos contra p-ANCA, en ausencia de granulomas extravasculares y depósitos de inmunocomplejos en la inmunofluorescencia directa. La poliangeitismicroscopica tiene un pronóstico peor que las otras formas de vasculitis asociadas a ANCAS con una supervivencia a los 5 años del $45 \%$. 


\section{Vasculitis de debut en la infancia}

Vol. 3, núm. 1., (2019)

Erika Teresa Tumbaco Macías; Johang Mauricio Olivo Román; Cesar Enrique Arreaga Pérez; Ronald Adrián Valencia Rodríguez

Otra importante vasculitis en la etapa de la infancia es la enfermedad de Kawasaki (EK) es una vasculitis frecuente que puede lesionar las arterias coronarias originando aneurismas, en ocasiones, se complican con trombosis u obstrucción coronaria provocando isquemia miocárdica1. Constituye la primera causa de cardiopatía adquirida en la infancia del adulto joven, desplazando a la Fiebre Reumática en los países desarrollados. Un diagnóstico oportuno y tratamiento adecuado durante la fase aguda de la enfermedad disminuyen el riesgo de coronariopatía. El uso de gammaglobulina parenteral en los primeros 10 días de la enfermedad disminuye este riesgo de un $20 \%$ a un $5-2 \%$. El compromiso coronario se presenta con mayor frecuencia en varones, menores de un año, con período de inflamación prolongada o recurrencia de la fiebre luego de un período a febril. También suelen hallarse si el paciente presenta signos de isquemia, afección miocárdica o descenso del nivel plaquetario, albúmina y hematocrito respecto al nivel inicial. 


\section{Vasculitis de debut en la infancia}

Vol. 3, núm. 1., (2019)

Erika Teresa Tumbaco Macías; Johang Mauricio Olivo Román; Cesar Enrique Arreaga Pérez; Ronald Adrián Valencia Rodríguez

Figuras: 1-2-3 y 4 Aspecto Físico de las Vasculitis en Niños

\section{INYECCION CONJUNTIVAL}

\section{CAMBIOS OROFARINGE}
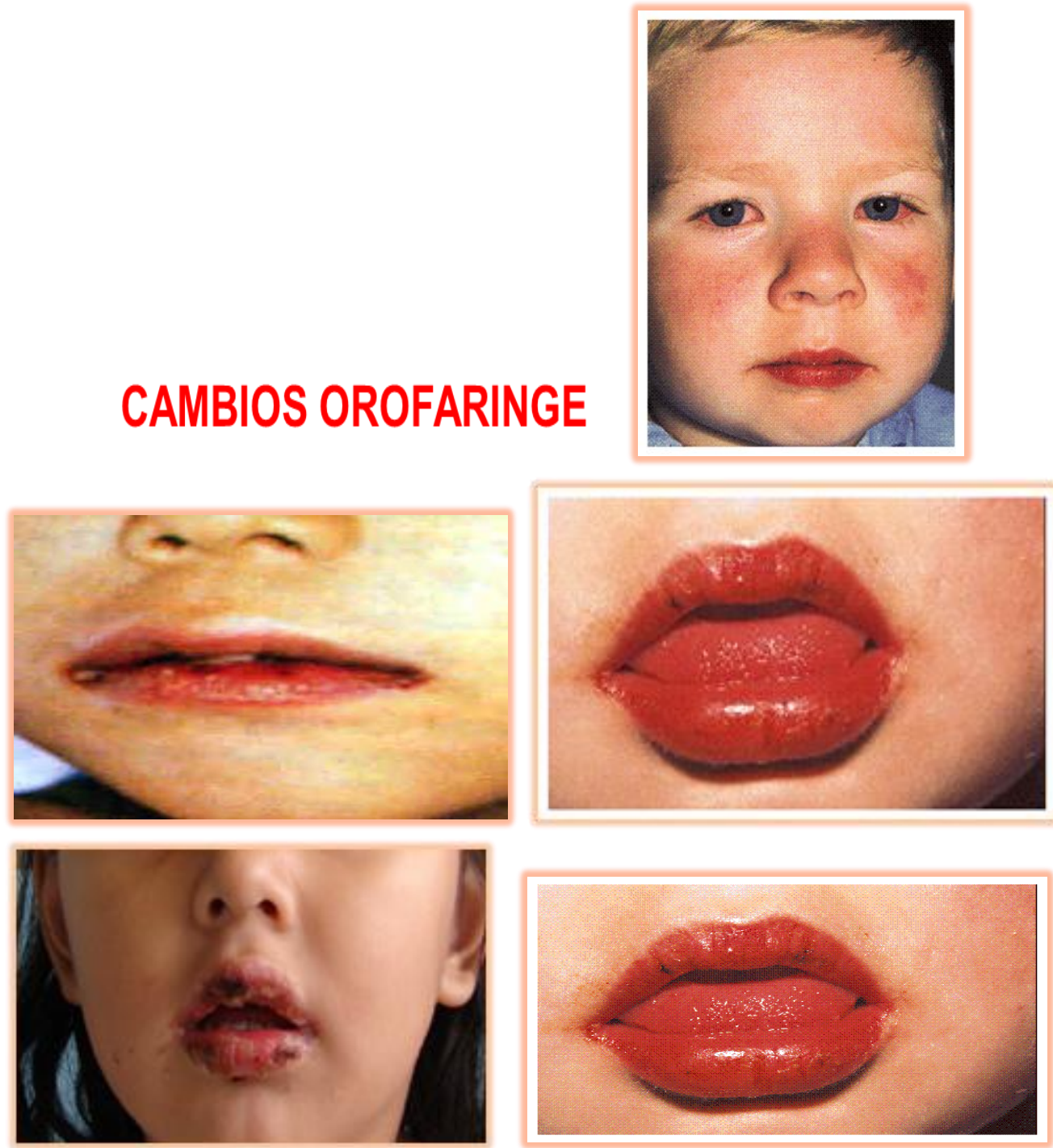

Fuente: Elaboración Propia (2019) 


\section{Vasculitis de debut en la infancia}

Vol. 3, núm. 1., (2019)

Erika Teresa Tumbaco Macías; Johang Mauricio Olivo Román; Cesar Enrique Arreaga Pérez; Ronald Adrián Valencia Rodríguez

\section{Conclusiones.}

El desarrollo de los diversos aspectos que fueron considerados para la presentación de este artículo, llevan a estimar una serie de argumentaciones reflexivas como expresión analítica que guarda vinculación con los diferentes aportes dados por aquellas fuentes teóricas secundarias revisadas para ampliar de manera precisa lo tópicos requeridos. Por ello, se concluye lo siguiente:

La vasculitis como enfermedad presenta diferentes características, signos y síntomas que conduce a las ciencias médicas, particularmente las relacionadas con el área de pediatría, buscar informaciones coherentes que les permitan llegar a un diagnóstico básico, para lo cual, necesitan una serie de exámenes clínicos, laboratorios, radiografías y por último la biopsia, pero la misma necesita de espacios grandes que logren extraer el material requerido para el estudio.

Es importante acotar que, la piel como órgano del cuerpo humano representa un importante reporte para esta enfermedad, donde que sus diferentes tipos se encuentran determinados por infecciones cutáneas que le pueden servir de referencia al especialista, pero al mismo tiempo ofrecen informaciones que pueden ser vinculadas con otras manifestaciones o enfermedades, de allí, la importancia del diagnóstico de forma precisa; pues, la inflamación de los vasos pequeños, medianos y grande llevan a estimar la presencia de diferentes agentes.

Cabe agregar, que las vasculitis debido a la inflamación y características heterogéneas que presentan llevan a configurar nuevos síndromes con particulares especiales que han sido estudiadas y se conocen sus efectos, sin embargo en la etapa de la infancia los casos son reducidos, existe la enfermedad de Kawasaki (EK), que representa una afección a nivel del 


\section{Vasculitis de debut en la infancia}

Vol. 3, núm. 1., (2019)

Erika Teresa Tumbaco Macías; Johang Mauricio Olivo Román; Cesar Enrique Arreaga Pérez; Ronald Adrián Valencia Rodríguez

corazón, demostrando que el compromiso coronario se evidencia con mayor tendencia en los varones.

\section{Bibliografía.}

Castellanos, H. (2018). Investigación Documental . México: Limusa.

González, R. (2015). Medidas de Prevención en Infecciones de Piel . Chile: Vergara.

La Cruz, J. y. (2014). Diagnóstico de la Vasculitis Sistémica . Barcelona: Paidós.

Medina, A. (2014). Patogenesis y Clasificación de las Vasculitis . La Habana: Medio Mundo.

Méndez, G. (2018). Metodología e Investigación. México: Mac Graw Hill.

Mogollón, N. (2019). Técnicas para la Investigación Documental . Barquisimeto: CIDEG.

Quintana, B. (2015). Vasculitis Sinstémica en Pediatría . España: Narcea. 